\section{Resolution in partially accomodative esotropia during occlusion treatment for amblyopia}

F Koc, H Ozal, H Yasar and E Firat
Purpose To evaluate alignment changes in partially accommodative esotropia during occlusion treatment for amblyopia.

Method Changes at the deviation angles of 63 partially accommodative esotropia patients, who had occlusion treatment for amblyopia, were evaluated retrospectively.

Results Mean deviation angle at the start of therapy without glasses was 45 PD (10-90 PD) and became 27 PD (5-70 PD) after at least 2 months with glasses. During 12 (2-36) months of occlusion period, mean manifest deviation angle with glasses decreased to 11 PD (0-50) $(P<0.001)$ and amblyopia resolved in $71.5 \%$ of the cases. After termination of amblyopia treatment $24(38 \%)$ cases had surgery for the residual deviation but if we had planned surgery before amblyopia treatment, $81 \%$ of the patients would have had surgery.

Discussion Should amblyopia be treated initially or should we operate first in patients with strabismus and amblyopia together? Our research suggests that we should not hurry to operate in high hypermetropic partially accommodative cases, which have amblyopia and a long-term history of strabismus. Initial amblyopia treatment in these cases allows time for resolution of the nonaccomodative component in strabismus and can significantly decrease the necessity for surgery.

Eye (2006) 20, 325-328. doi:10.1038/sj.eye.6701874; published online 3 June 2005

Keywords: amblyopia; accomodation; esotropia; occlusion treatment; surgery

\section{Introduction}

An esotropia is partially accommodative when accommodative factors contribute to but do not account for the entire deviation. ${ }^{1}$ Accomodative components are usually expected to resolve after 6-8 weeks of full correction. Partially accommodative esotropia can develop secondary to accommodative esotropia, due to contraction in muscles conjunctiva and tenons capsule, if the treatment is delayed. ${ }^{1,2}$ Treatment would then be surgery for the residual deviation after correction for the accommodative component with refraction. Amblyopia usually complicates partially accommodative esotropia. In this study, we attempt to answer: should we operate first or treat amblyopia in partially accommodative esotropia.

\section{Materials and methods}

The records of patients who had occlusion treatment for amblyopia were reviewed retrospectively, in a search for cases meeting specific inclusion criteria for this study. These parameters were: (1) having hypermetropia $\geq 1.5 \mathrm{dpt}$; (2) presence of residual esotropia in spite of full correction at least 2 months; (3) presence of amblyopia (at least two line difference in Snellen's chart or fixation preference); (4) having occlusion treatment; (5) being less than $\leq 8$ years of age; and (6) no evidence of central nervous system abnormalities or other anatomic anomalies of the eyes that might affect normal visual development. In all, 63 patients met the criteria for inclusion in this study. Data collected from the medical records included gender, age, spherical equivalents of refractive errors, age at
Strabismus, SSK Ankara Eye Disease Hospital, Ulucanlar, Ankara, Turkey

Correspondence: F Koc, Kuzgun Sok 48/3, Asagi Ayranci 06540, Ankara, Turkey

Tel: + 9031246874 59;

Fax: + 903123124827 .

E-mail: dr_feray@

yahoo.com

Received: 11 November 2004

Accepted: 15 February 2005 Published online: 3 June 2005 
onset of esotropia, age at the start of treatment, duration of esodeviation, distant manifest deviations; at the onset of therapy, after two months wearing glasses and at the end of occlusion treatment, duration of amblyopia treatment and success rate, frequency of surgery, motor and sensory fusion frequencies at the end of therapy, and length of follow-up.

Visual acuities were tested by Snellen's chart or ' $E$ ' chart in verbal children and by fixation preference method in preverbal children. After cylcoplegic refraction with cylopentolate or tropicamide, fullcorrecting glasses were prescribed to the patients. Duration of occlusion and frequencies of follow-up were determined according to the patient's age and response to treatment.

Initial suggested occlusion durations were half of the awakened hours (2-6h). Occlusion duration was increased in resistant cases. Deviation angles were measured with prism-cover test, if not possible, with Krimsky method at each visit. Distant manifest deviation angles with glasses before and after occlusion treatment were compared with Paired-Sample $T$ test. After resolution of amblyopia, surgery was applied if the patients still have manifest deviations with glasses. In cases where the patient had resistant amblyopia, surgery was applied after at least 6 months of amblyopia treatment trial, if they had stable deviations. Binocular functions were evaluated by Worth four-dot and Titmus Fly tests.

\section{Results}

Table 1 provides descriptive data on the 63 patients in this study group. Most of the patients were high hypermetropic and five patients had also high accommodative convergence - accomodation ratio. For the treatment of amblyopia, occlusion treatment was applied 2-36 months of duration (mean; 12.19 \pm 8.93 ). This duration included noncompliant periods to occlusion treatment also. All patients continued to have 2 hours maintenance occlusion after resolution of amblyopia.

We have observed progressive decrease in the deviation angles of most of the cases. Figure 1 summarizes deviation changes throughout follow-up period. Table 2 shows mean manifest deviation angles with glasses prior to and after treatment. It was $27 \mathrm{PD}$ (5-70) at the start of amblyopia treatment, with a significant decrease $(P<0.001)$ during occlusion period, it became $11 \mathrm{PD}(0-50)$. Deviation angles were taken as 0 for the patients who were orthophoric with glasses at the end of occlusion treatment while calculating the mean, since latent deviations had not been measured in these cases.
Table 1 Patient data

\begin{tabular}{lc}
\hline Number of patients & 63 \\
Sex (F/M) & $31 / 32$ \\
Age at onset of strabismus (year) & $2.59 \pm 1.56$ \\
Age at onset of treatment (year) & $4.50 \pm 1.84$ \\
Age at orthophoria attained (year) & $5.96 \pm 2.03$ \\
Duration of deviation (year) & $3.38 \pm 1.77$ \\
Refractive error (ambliyopic & $4.28 \pm 1.74 / 4.92 \pm 1.74$ \\
eye/normal eye) & \\
Follow-up period (months) & $48.62 \pm 24.51$ \\
\hline
\end{tabular}

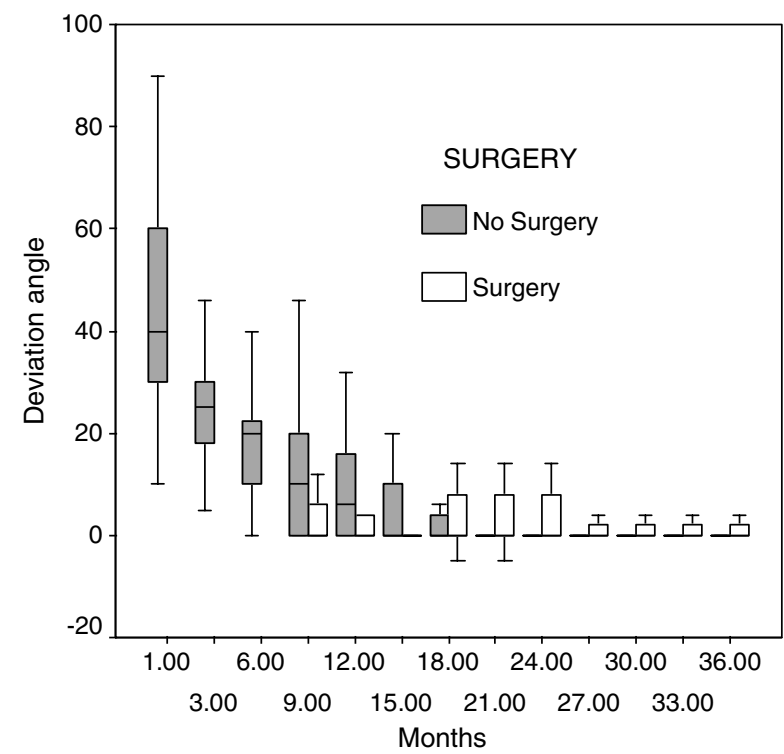

Figure 1 Changes in deviation angles during occlusion period.

Table 2 Deviation angles and percentages of patients at the surgical limits prior to and after occlusion period

\begin{tabular}{|c|c|c|c|}
\hline & $\begin{array}{l}\text { Prior to } \\
\text { treatment }\end{array}$ & $\begin{array}{c}\text { After } \\
\text { treatment }\end{array}$ & $\mathrm{P}$ \\
\hline $\begin{array}{l}\text { Manifest deviation } \\
\text { angle with glasses } \\
\text { (PD) }\end{array}$ & $27.09 \pm 15.07$ & $11.31 \pm 14.23$ & $<0.001$ \\
\hline $\begin{array}{l}\text { Number of patients } \\
\text { at surgical limits } \\
(\geq 12 \text { PD) }\end{array}$ & $51(81 \%)$ & $24(38 \%)$ & $<0.001$ \\
\hline
\end{tabular}

Amblyopia resolved completely in $71 \%$ of the cases and the depth of it decreased in other cases. Surgery was applied after resolution of amblyopia if there was still stable deviation with glasses. In resistant amblyopia cases surgery was applied if they had stable deviation after at least 6 months of occlusion treatment. If we had planned surgery before amblyopic treatment, $81 \%$ of the patients would have had deviations within surgical limits ( $\geq 12$ PD), but after amblyopia treatment only $38 \%$ needed surgery $(P<0.001)$. Eventually, all patients were 
Table 3 Outcomes for the study group

\begin{tabular}{lc}
\hline & Success rate (\%) \\
\hline Amblyopia treatment & 71 \\
Motor success $(\leq \pm 10 \mathrm{PD})$ & 100 \\
${\text { Fusion }(\mathrm{W} 4 \mathrm{D})^{\mathrm{a}}}_{\text {Stereopsis }^{\mathrm{a}}}$ & 79.5 \\
\hline
\end{tabular}

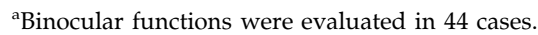

orthophoric ( \pm 10 PD) and stayed ortophoric throughout the follow-up period of 4 years ( 7 months to 9 years). Binocular functions were evaluated in 44 patients: 35 patients $(79.5 \%)$ had fusion at distance or only at near with Worth four-dot test and 26 patients (59\%) had stereopsis changing 40-3000 arcs. Table 3 presents the main outcomes of the study.

\section{Discussion}

To achieve a stable outcome in strabismus surgery, patients should be prepared for fusion before surgery. For sensory fusion to occur, the images not only must be located on corresponding retinal areas but also must be sufficiently similar in size, brightness, and sharpness. ${ }^{3}$

The case of unequal images, as in amblyopia, is a severe sensory obstacle to fusion. The aim of amblyopia treatment is to provide similar images to the correspondent retinal points, whereas the aim of strabismus surgery is to decrease the deviation within the limits of individual motor fusion amplitudes. Classical teaching recommends treating amblyopia first, but recently this approach has come under dispute. Surgeons disputing classical teaching have begun to initiate surgery earlier to mitigate the psychological effects on the patient and their family of having strabismus for an extended period of time. In these studies, they also claim that early surgery does not change the motor and sensory results except in infantile esotropia cases. ${ }^{4-6}$ In our study, we claim that amblyopia treatment should be the initial treatment in partially accommodative cases where evidence of amblyoia also exists. We have observed a progressive decrease in the deviation angles of partially accommodative esotropia cases, which had occlusion treatment for their amblyopia. Mean deviation angle with glasses was $27.09 \pm 15.07$ PD at the start of occlusion treatment. Our results indicate a significant decrease during occlusion treatment $(P<0.001)$, to a level of $11.31 \pm 14.23$ PD at the end of occlusion treatment. If we had operated on the patients first, $81 \%$ of them would have had deviations within surgical limits (12-70 PD). At the end of occlusion treatment, this rate showed a significant decrease $(P<0.001)$ with only $38 \%$ of them requiring surgery.
The effects of occlusion can vary widely. It can convert phoria to tropia by causing the loss of the ability of fusion. Holbach et $a l^{7}$ found that the deviation changes during occlusion treatment of strabismic amblyopic cases were not much different from the fluctuation of the deviations in nonamblyopic strabismic cases in a group of nonhomegeneous esotropia patients after 3 months (1-13 months) of follow-up period. In all, 22 of Holbach's patients were infantile esotropes and the remainder were not classified. They also did not mention the refractive status of their patients. Our study focused specifically on partially accommodative cases usually with high hypermetropia (mean hypermetropia in the normal eye; $4.28 \mathrm{dpt} /$ mean hypermetropia in the amblyopic eye; $4.92 \mathrm{dpt}$ ). Additionally, our group had a mean of 2 years of esotropia history before the start of the therapy, which we thought to be the main reason of nonaccomodative components in most of the patients. We evaluated angular changes for a period of 12 months (2-36 months). Although we observed a significant decrease in the deviation angles of our patients, we encountered a weak point, which caused the overestimation of these changes in some cases. To explain, we did not apply the prism and cover test in patients who reached othophoria with the cover test. This resulted in an ignorance of any latent deviations in these patients.

Delay in the search for treatment in accommodative esotropia results in the development of amblyopia and the development of a nonaccomodative component in esotropia. Our patients had a mean of 2 years delay for the initiation of therapy. We believe that it is not the occlusion treatment itself, but instead the time spent for this treatment, which allows the time for the resolution of the nonaccomodative component in partially accommodative esotropia. Full refractive correction decreases the convergence tonus, coming to a new equilibrium of extraocular muscles and surrounding tissue might be time consuming. As the amount of deviation decreases and the amblyopia becomes resolved, the patient may regain fusional divergence and turn the manifest deviation into a latent phase. Since the onset of strabismus occurred after the critical period for binocular development, our group was expected to have the advantage of high binocular potential. Thus, $79.5 \%$ of the patients who had binocular tests demonstrated binocular functions at different levels.

Initial surgery in these cases might have resulted in late consecutive exotropia. As is already known, the presence of amblyopia ${ }^{8}$ and hypermetropia ${ }^{9}$ are the most common risk factors for the development of consecutive exotropia and are also typical characteristics of most of the partially accommodative esotropia cases. Another risk factor for the development of consecutive exotropia is postoperative underaction of medial rectus ${ }^{8}$ for which 
high hypermetropic partially accommodative cases are also more prone with their smaller globe size. Although our 4-year follow-up period could be considered relatively short to draw these conclusions, this conservative approach might be a preventative factor for the development of consecutive exotropia. In spite of the fact that our results indicated nearly $30 \%$ amblyopia in a high hypermetropic population, we evidenced no consecutive exotropia. In long-term follow-up, the rate of consecutive exotropia was recorded as high as $20 \%$ in esotropic cases. ${ }^{10}$

In conclusion, our study suggests that, we should not hurry to operate on high hypermetropic partially accommodative cases, which have amblyopia and a longterm history of strabismus. Initial amblyopia treatment in these cases allows time for the resolution of the nonaccomodative component in strabismus.

\section{References}

1 Von Noorden G. Esodeviations. In: Von Noorden G (ed). Binocular Vision and Ocular Motility, 5th ed. C.V. Mosby: St Louis, 1996, pp 303-308.
2 Koc F, Ozal H, Kargi S, Firat E. Clinical evaluation of pure and partial accommodative esotropia. Turk Oftalmoloji Gazetesi 2000; 30: 381-386.

3 Von Noorden G. Binocular vision and space perception. In: Von Noorden G (ed). Binocular Vision and Ocular Motility, 5th ed. C.V. Mosby: St Louis, 1996, pp 8-40.

4 Subhash D, Kamlesh MS. Is it mandatory to treat amblyopia prior to surgery? Acta Opthalmol Scand 2001; 79: 28-30.

5 Lam GC, Repka MX, Guyton DL. Timing of amblyopia therapy relative to strabismus surgery. Opthalmology 1993; 100(12): 1751-1756.

6 Weakley DR, Holland DR. Effect of ongoing treatment of amblyopia on surgical outcome in esotropia. J Pediatr Opthalmol 1997; 34(5): 275-278.

7 Holbach HT, Von Noorden GK, Avilla CW. Changes in esotropia after occlusion therapy in patients with strabismic amblyopia. J Pediatr Ofthalmol 1991; 28(1): 6-9.

8 Folk ER, Miller MT, Chapman L. Consecutive exotropia following surgery. Br J Ophtalmol 1983; 67: 546-548.

9 Kushner BJ. Partly accomodative esotropia. Should you overcorrect and cut the plus? Arch Opthalmol 1995; 113: 1530-1534.

10 Dunnington JH, Regan EF. Factors influencing the postoperative result in concomitant convergent strabismus. Arch Opthalmol 1950; 44: 813-822. 